Draft VERSION OCTOBER 20, 2021

Typeset using LATEX twocolumn style in AASTeX631

\title{
Transient phenomena from gas erupting neutron stars
}

\author{
Huiquan Li ${ }^{1,2,3}$ AND JiAnChEng WANG ${ }^{1,2,3}$ \\ ${ }^{1}$ Yunnan Observatories, Chinese Academy of Sciences, \\ 650216 Kunming, China \\ ${ }^{2}$ Key Laboratory for the Structure and Evolution of Celestial Objects, \\ Chinese Academy of Sciences, 650216 Kunming, China \\ ${ }^{3}$ Center for Astronomical Mega-Science, Chinese Academy of Sciences, \\ 100012 Beijing, China
}

\begin{abstract}
Starquakes probably occur in rapidly spinning or ultra high field neutron stars. In this article, we argue that highly compressed gas containing electron-positron pairs could evaporate and erupt from inside the neutron star when a crack forms and then heals during a starquake. Under the influence of the existing oscillation modes of the star, the crack may have sufficiently large size and long lifetime. An appropriate amount of gas can erupt into the magnetosphere with relativistic and nearly uniform velocity, producing various transient and bursting phenomena.
\end{abstract}

\section{INTRODUCTION}

Starquake due to crustal failure probably occurs in neutron stars that spin fast or have strong fields (e.g., Levin \& Lyutikov (2012)). In the outer envelopes of these objects, the shear stress accumulates until the crust cracks. Starquakes can naturally lead to glitches observed in some pulsars (Ruderman 1969, 1991; Chamel \& Haensel 2008). In the popular models, the intense crustal fractures cause high energy flares from magnetars (Thompson \& Duncan 1995) and the smallscale fractures lead to the persistent X-ray emission (Thompson \& Duncan 1996).

However, regarding the fracture model, there remain some doubts. It is argued by (Jones 2003) and further tested in molecular simulations (Horowitz \& Kadau 2009) that no voids or fractures can occur due to the tremendous isotropic pressure in the crust. The voids can only exist in extremely short time and heal very quickly. In magnetars, the strong magnetic fields will impede the shear motion and so will strongly limit the energy released from the crack of the crust (Levin \& Lyutikov 2012). Indeed, the analysis should be applicable to the ideal and simple case. But, there may still exist mechanisms that can create larger voids or cracks with a longer lifetime in a realistic neutron star under some complex circumstances. For example, when the fracture of the crust is coupled with the existing oscillation modes, the forming crack may be amplified.

In this work, it is pointed out that the crustal cracks might lead to gas eruption from the neutron star. Here, we only care about the size and lifetime of the voids or cracks instead of the intensities and other properties of the fractures. We keep open on these quantities of the cracks: they may be very small as anticipated by the works (Jones 2003; Horowitz \& Kadau 2009) or somehow larger due to some unknown mechanisms. Once a crack forms, it will be quickly filled with gas that comes from the highly compressed crust or is created locally. The filled gas will later be compressed and expelled from the crack as the defect heals, producing transient emission in the magnetosphere. The neutral gas may contain electro-positron pairs, free neutrons and other plasma of light elements from inside the crust and the atmosphere. We show that the energy taken away by the erupted gas can reach the ones of giant pulses (GPs) and fast radio bursts (FRBs) if the size and lifetime of the cracks are large enough. This model is different from the neutron star volcano model (Dyson 1969) where baryonic lava erupt into the interstellar region from the interior of the neutron star.

\section{ERUPTION OF GAS FROM NEUTRON STARS}

Once a crack forms as the stress accumulates in the crust, the pressure in the vacuum crack will suddenly become zero. It will be quickly filled up with the gas of light, relativistic particles coming from the highly compressed crust and atmosphere. The characteristic speed of propagation of the cracks is (Thompson \& Duncan 1995)

$$
c_{s}=\sqrt{\mu / \rho} \simeq 1.4 \times 10^{8} \mathrm{~cm} \cdot \mathrm{s}^{-1},
$$

where $\mu$ is the shear modulus and $\rho$ is the mass density. The speed of the relativistic gas is nearly the speed of 
light. So there should be enough time for the gas to fill the crack and erupt from it before the crack heals. We think that the gas contains the following possible components.

First, the electron-positron pairs. In a young neutron star, electron-positron pairs can be produced in the presence of high temperature and magnetic fields. Particularly for a magnetar, the magnetic field is ultra strong $>10^{15} \mathrm{G}$, much larger than the Schwinger limit. So the pairs can be created via the one-photon and the Schwinger processes. On the other hand, the pairs can annihilate into photons and neutrinos. The latter is one of the key mechanism that the energy is taken away from neutron star so that a young star can cool down (Chiu \& Morrison 1960; Yakovlev et al. 2001; Chamel \& Haensel 2008). In the equilibrium state, the creation rate and the annihilation rate of the pairs should be equal.

The density of the pairs is highest in the low-density and high-temperature plasma (Yakovlev et al. 2001; Chamel \& Haensel 2008). In the crack, the matter density is zero and there are no degenerate electrons. When a crack forms, the highly compressed pairs in the crust can drift into it from nearby regions. Moreover, extra pairs can be created in the crack simultaneously in the presence of high temperature and magnetic fields. The pairs should be in the relativistic regime, which makes them not easy to annihilate. Besides, the strong magnetic fields should also reduce the annihilation rate with quantized motion. So it is possible that the crack is filled with high density pairs.

Second, the gas or liquid in the atmosphere and magnetosphere will be possibly sucked into the crack as the vacuum crack forms. This component of plasma is strongly influenced by the magnetic fields. Only the plasma near the exit of the crack can slide into it along the magnetic field lines. The plasma contains light elements, like hydrogen and helium, and electro-positron plasma.

Finally, free neutrons. Deep into the inner crust with increasing density and pressure, the free neutrons enrich. The free neutrons are light and neutral. So they can quickly evaporate into the crack if the pressure is suddenly zero. The neutrons are much heavier than electrons and they should be minor in the gas.

When the crack forms, it will be loaded with these components of the gas quickly. When the crack heals, the gas will be expelled out by the isotropic pressure of the crust. Since the pressure in the crust is huge, we expect that the erupted gas attains extremely relativis- tic velocity. Their velocities should be nearly uniform ${ }^{1}$ since they are expelled by an almost constant pressure. The Lorentz factors of the gaseous particles depend on the energy budget and the component, amount of the particles.

The maximum energy that the gas possibly attains is of the scale:

$$
E=P v
$$

When the density $\rho$ varies from $10^{4} \mathrm{~g} \cdot \mathrm{cm}^{-3}$ to $10^{14}$ $\mathrm{g} \cdot \mathrm{cm}^{-3}$ in the crust, the average pressure is in the range $10^{19} \mathrm{dyn} \cdot \mathrm{cm}^{-2}<P<10^{33} \mathrm{dyn} \cdot \mathrm{cm}^{-2}$ (e.g., see Haensel et al. (2007); Chamel \& Haensel (2008)). Thus, to release large enough energy, the volume $v$ of the crack is the key in this model. Another important factor is the lifetime of the crack. A large enough lifetime is necessary for the gas to have enough time to escape from the bottom of the crack. More importantly, in a longer lifetime, the crack is loaded with more gas. More electronpositron pairs are created and present.

\section{THE SIZE AND LIFETIME OF A CRACK}

The volume of the erupted gas can be estimated by that of the crack. We express the latter as

$$
v=l d \delta
$$

where $l, d$ and $\delta$ are respectively the length, thickness and width of the crack. Following the fracture model (Thompson \& Duncan 1995), the length $l$ can be taken to be about $1 \mathrm{~km}$. The thickness should be the scale of that of the crust, i.e., also $d \sim 1 \mathrm{~km}$. Thus, the lifetime of the crack should be larger than $d / c \sim 10^{-5}$ s so that the gas at the bottom of the inner curst has enough time to come out. In the fracture model (Thompson \& Duncan 1995), the shear displacement is about $100 \mathrm{~m}$ and the crack lifetime is $\sim 100 \mathrm{~m} / c_{s} \sim 10^{-4} \mathrm{~s}$. So the gas can escape in time in the model.

However, as analyzed in (Jones 2003), the width of the crack is several times of the mean distance between ions $\left(\delta \sim 10^{-11} \mathrm{~cm}\right)$ since the isotropic pressure in the crust is huge. So it has an extremely short lifetime $\sim 10^{-19} \mathrm{~s}$. Then the energy taken away by the gas is quite negligible in our model, following the arguments. For such a short lifetime, the fracture model (Thompson \& Duncan 1995, 1996) also does not work because the shear motion will stop instantly.

But, this might be not necessarily true in some special cases. For example, the isotropic pressure may be not

\footnotetext{
${ }^{1}$ They should be uniform both in speed and in direction. The gas should be ejected at almost the same direction, with a jet-like structure.
} 
so strong temporally when the crack happens on a fast spinning neutron star or a binary system. The pressure is a total effect of the gravitational and centrifugal forces on the crust. For rapidly spinning neutron stars, the centrifugal force is comparable to the gravitational force. At the moment of fracture, the gravitational force on the broken tectonic plate can be partially balanced by the centrifugal force. This may make the crack live longer.

Here, we are more interested in another case. When the fracture of the crust is coupled with the oscillation modes that are propagating in the star, the wound of a crack may be amplified. It is known that neutron stars can sustain various oscillation modes (van Horn 1980; McDermott et al. 1988). Some of the modes are left behind after the birth of the star. The energy stored in an oscillation mode is about $\sim 10^{50}$ ergs (Thorne 1969; McDermott et al. 1988).

As the non-radial oscillation modes that can cause the variation of density and pressure, the lowest level pmodes have periods of the scale $\sim$ ms (Thorne 1969; McDermott et al. 1988). When the oscillating crust cracks suddenly, its dynamics should be strongly influenced by the varying pressure of the modes. If the fracture happens to occur near the trough of the dominated wave, the broken tectonic plates on both sides of the crack will be stretched in opposite directions. Then the crack may be made wider. It should have a longer lifetime, close to the period of the mode. The oscillating modes do not change the total energy and angular momentum transfer between different parts of the star. So the glitch behaviour should remain unchanged though the crack is amplified.

If the width of the amplified crack reaches $\delta \sim 1 \mathrm{~cm}$, then the energy budget for the erupted gas is

$$
E=10^{40} \frac{P}{10^{30} \mathrm{dyn} \cdot \mathrm{cm}^{-2}} \frac{l}{1 \mathrm{~km}} \frac{d}{1 \mathrm{~km}} \frac{\delta}{1 \mathrm{~cm}} \mathrm{ergs},
$$

where we choose the typical pressure of the inner crust $10^{30} \mathrm{dyn} \cdot \mathrm{cm}^{-2}$. This energy is much less than the oscillation energy of the star.

\section{TRANSIENT PHENOMENA}

The free neutrons in the erupted gas are expected to go through the magnetosphere without interactions since their lifetime is nearly 15 minutes. They may produce observable effect when they reach the outer medium, where they decay into protons and electrons. But, the charged particles in the erupted gas will play significant role in the magnetosphere. The abrupt and short-duration eruption of electron-positron pairs from the neutron star may be responsible for the transient phenomena observed from magnetars and fast spinning neutron stars.
If the charged particles erupt in the open magnetic field line region with nearly uniform and highly relativistic velocities, they will slide along the field lines and escape to the far regions. During the process, the relativistic charges can initiate pair cascade and create a large amount of pairs additionally. When they move out to the outer regions where the field becomes weaker and the cascade mechanism does not effectively work, they produce beamed and coherent radio emission, which may account for the GPs and FRBs. The detailed process is quite similar to the ejection and radiation process of electron-positron pairs discussed in the model of Geng et al. (2021), which is due to the collapse of the accumulated material accreted from the companion star in the polar region.

The GPs are very intense and short-duration radio pulses with respect to average pulses (see Kuzmin (2007); Eilek \& Hankins (2016) for reviews). Our gaserupting model may explain the first class of GPs observed from some young and rapidly spinning neutron stars (Sun et al. 2021). The GPs are 2 to 4 orders stronger than average pulses and have timescale of nanoseconds to microseconds. So smaller size or shorter lifetime of the crack than that given in Eq. (4) is needed to explain the GPs. They may be produced by smaller-scale but more frequent fractures of the crust (Thompson \& Duncan 1996).

FRBs (Lorimer et al. 2007) are among the most puzzling transient phenomena. In a millisecond duration, the characteristic energy $10^{35}-10^{43}$ ergs can be released during a burst. Their origin from magnetars is preferred nowadays (CHIME/FRB Collaboration et al. 2020; Bochenek et al. 2020; Zhang 2020). FRBs take similarities to GPs (Lyu et al. 2021) and can be viewed as super-GPs from young and rapidly spinning pulsars (Cordes \& Wasserman 2016; Katz 2016; Lyutikov et al. 2016). Previously, Wang et al. (2018) revealed evidence indicating that the repeating FRBs originate from starquake induced behaviour. Recent work (Yang \& Zhang 2021) based on the fracture model of magnetars is supposed to explain the associated emission of an FRB at the radio and X-ray band. Here, we provide an alternative starquake model to interpret the FRB phenomena: they may be explained as a result of gas eruption from magnetars. Our model does not require intense fracture of the crust. We only need the crack to have a large enough volume and lifetime. The typical FRB energy is reachable as a crack forms with the quantities comparable to those given in Eq. (4).

If the charged particles erupt in the closed field line region, they will be trapped in this region. Since the curvature of the field lines is larger in this region, high 
may be relevant to the high energy bursts and flares observed from some magnetars.

\section{REFERENCES}

Bochenek, C. D., Ravi, V., Belov, K. V., et al. 2020, Nature, 587, 59, doi: 10.1038/s41586-020-2872-x

Chamel, N., \& Haensel, P. 2008, Living Reviews in Relativity, 11, 10, doi: 10.12942/lrr-2008-10

CHIME/FRB Collaboration, Andersen, B. C., Bandura, K. M., et al. 2020, Nature, 587, 54, doi: 10.1038/s41586-020-2863-y

Chiu, H.-Y., \& Morrison, P. 1960, PhRvL, 5, 573, doi: 10.1103/PhysRevLett.5.573

Cordes, J. M., \& Wasserman, I. 2016, MNRAS, 457, 232, doi: $10.1093 / \mathrm{mnras} / \mathrm{stv} 2948$

Dyson, F. J. 1969, Nature, 223, 486, doi: 10.1038/223486a0

Eilek, J. A., \& Hankins, T. H. 2016, Journal of Plasma Physics, 82, 635820302, doi: 10.1017/S002237781600043X

Geng, J., Li, B., \& Huang, Y. 2021, The Innovation, 2, 100152, doi: 10.1016/j.xinn.2021.100152

Haensel, P., Potekhin, A. Y., \& Yakovlev, D. G. 2007, Neutron stars 1: Equation of state and structure, Vol. 326 (New York, USA: Springer), doi: 10.1007/978-0-387-47301-7

Horowitz, C. J., \& Kadau, K. 2009, PhRvL, 102, 191102, doi: 10.1103/PhysRevLett.102.191102

Jones, P. B. 2003, ApJ, 595, 342, doi: 10.1086/377351

Katz, J. I. 2016, ApJ, 818, 19, doi: 10.3847/0004-637X/818/1/19

Kuzmin, A. D. 2007, Ap\&SS, 308, 563, doi: 10.1007/s10509-007-9347-5

Levin, Y., \& Lyutikov, M. 2012, MNRAS, 427, 1574, doi: 10.1111/j.1365-2966.2012.22016.x
Lorimer, D. R., Bailes, M., McLaughlin, M. A., Narkevic, D. J., \& Crawford, F. 2007, Science, 318, 777, doi: 10.1126/science.1147532

Lyu, F., Meng, Y.-Z., Tang, Z.-F., et al. 2021, Frontiers of Physics, 16, 24503, doi: 10.1007/s11467-020-1039-4

Lyutikov, M., Burzawa, L., \& Popov, S. B. 2016, MNRAS, 462, 941, doi: 10.1093/mnras/stw1669

McDermott, P. N., van Horn, H. M., \& Hansen, C. J. 1988, ApJ, 325, 725, doi: 10.1086/166044

Ruderman, M. 1969, Nature, 223, 597, doi: 10.1038/223597b0

—. 1991, ApJ, 382, 587, doi: 10.1086/170745

Sun, S. N., Yan, W. M., \& Wang, N. 2021, MNRAS, 501, 3900, doi: 10.1093/mnras/staa3825

Thompson, C., \& Duncan, R. C. 1995, MNRAS, 275, 255, doi: $10.1093 / \mathrm{mnras} / 275.2 .255$

—. 1996, ApJ, 473, 322, doi: 10.1086/178147

Thorne, K. S. 1969, ApJ, 158, 1, doi: 10.1086/150168

van Horn, H. M. 1980, ApJ, 236, 899, doi: 10.1086/157816

Wang, W., Luo, R., Yue, H., et al. 2018, ApJ, 852, 140, doi: 10.3847/1538-4357/aaa025

Yakovlev, D. G., Kaminker, A. D., Gnedin, O. Y., \& Haensel, P. 2001, PhR, 354, 1, doi: 10.1016/S0370-1573(00)00131-9

Yang, Y.-P., \& Zhang, B. 2021, arXiv e-prints, arXiv:2104.01925. https://arxiv.org/abs/2104.01925

Zhang, B. 2020, Nature, 587, 45, doi: $10.1038 /$ s41586-020-2828-1 